Coll, M. y Pérez Pucci, C. (2021). La producción lexicográfica de fines

del siglo XX en Uruguay

RILEX. Revista sobre investigaciones léxicas, 4/I, pp. 69-96.

\title{
LA PRODUCCIÓN LEXICOGRÁFICA DE FINES DEL SIGLO XX EN URUGUAY ${ }^{1}$
}

\section{LEXICOGRAPHY PRODUCTION IN THE LATE 2OTH CENTURY IN URUGUAY}

\author{
Magdalena Coll \\ Universidad de la República \\ collmagdalena@gmail.com \\ Clara Pérez Pucci \\ Universidad de la República \\ claraperezpucci@hotmail.com
}

\begin{abstract}
RESUMEN
En la segunda mitad del siglo xx se dio en Uruguay una producción lexicográfica vinculada, en distinta medida, con la actividad de la Academia Nacional de Letras del Uruguay (ANL). Se trata de seis obras de carácter diferencial con respecto al diccionario de la Real Academia Española (RAE), que, en su mayoría, han recibido escasa o nula atención por parte de la comunidad académica. De la descripción y análisis de estas obras trata, precisamente, este artículo que busca visualizar tendencias de la lexicografía uruguaya de fines del siglo Xx. Se ha estudiado cuál fue el origen de estas obras (muchas de ellas promovidas por concursos de la ANL o de la RAE), cuál era la formación de sus autores y autoras, cuál era su vínculo formal con la ANL y cuál fue la participación de las mujeres en esta etapa de la lexicografía uruguaya. También se ha tenido en cuenta si se trata de trabajos inéditos o de obras que lograron ser publicadas en Uruguay o en el exterior al tiempo que se analizó si tuvieron o no repercusión en el diccionario de la RAE. De la investigación realizada se destaca, entre otras cosas, que, aunque estas obras tienen originariamente una finalidad diferencial, pasan a ser valiosas en sí mismas porque, en su afán contrastivo, aportan a la descripción léxica del español del Uruguay de una época.
\end{abstract}

Palabras clave: Uruguay, siglo xx, lexicografía, tendencias.

\begin{abstract}
In the second half of the 20th century, the lexicographic production in Uruguay was linked, to varying degrees, to the activity of the Academia Nacional de Letras del Uruguay (ANL). Six works were written that contrasted with the RAE dictionary (DRAE) and which, for the most part, received little or no attention from the academic community. The description and analysis of these works is precisely the subject of this article, which seeks to visualize trends in Uruguayan lexicography at the end of the 20th century. We have studied the origin of these works (many of them promoted by ANL Or RAE contests), the academic training of their authors, their formal link with the ANL, and the participation of women in this stage of Uruguayan lexicography. It was also taken into account whether they were unpublished works or works that managed to be published in Uruguay or abroad, while analyzing whether or not they had an impact on the DRAE. From the research carried out, it is highlighted, among other things, that although these works originally have a contrastive purpose, they become valuable in themselves because, in their contrastive zeal, they contribute to the lexical description of the Uruguayan Spanish of an epoch.
\end{abstract}

Keywords: Uruguay, 20th century, lexicography, trends.

\footnotetext{
${ }^{1}$ Este trabajo fue realizado en el marco del proyecto "130 años de diccionarios: análisis conceptual y documental de la historia de la lexicografía uruguaya", que data de 2013. Se trata de una de las líneas actuales del Programa de Investigación en Terminología, Lexicografía especializada y Organización del Conocimiento (Programa TERM-OC), financiado por el programa Grupos de Investigación (2019-2023), de la Comisión Científica de la Universidad de la República, Uruguay, y coordinado por Barité y Coll. En ese contexto, se ha desarrollado la base "Daniel Granada", de fundamental importancia para la investigación que aquí presentamos. La caracterización de esa base se expone, más adelante, en este artículo.
} 


\section{SECCIÓN: LEXICOGRAFÍA \\ LA PRODUCCIÓN LEXICOGRÁFICA DE FINES DEL SIGLO XX EN URUGUAY \\ Magdalena Coll y Clara Pérez Pucci}

\section{PRESENTACIÓN}

Nos proponemos aquí describir la producción lexicográfica de la segunda mitad del siglo xx en Uruguay a partir de sus características más sobresalientes. Las obras que analizamos son de muy diferente índole, pero tienen como denominador común su fuerte vinculación con la actividad realizada en o promovida por la Academia Nacional de Letras del Uruguay (ANL) y su intención de completar, corregir o ampliar el Diccionario de la lengua española (DRAE) ${ }^{2}$ de la Real Academia Española (RAE). Se trata de seis obras lexicográficas, escritas entre 1963 y 1993, cuyo análisis nos permitirá delinear las tendencias de la lexicografía uruguaya de fines del siglo XX.

Después de los antecedentes (apartado 2), presentamos la metodología y los criterios de confección del corpus a analizar (apartado 3). El apartado 4 se centra en el análisis propiamente dicho de las obras seleccionadas. Las consideraciones finales, como es de uso, cierran este artículo.

\section{ANTECEDENTES}

Aun cuando un balance de la (meta)lexicografía en el Uruguay es tarea todavía pendiente, parece claro que habrá que empezar, siguiendo un criterio cronológico, por ver cómo el naturalista Félix de Azara ha sido estudiado como pionero lexicógrafo por Kühl de Mones (1997), Enguita Utrilla (2012) y Bértola (2013), al tiempo que los primeros escritores orientales, José Manuel Pérez Castellano y Dámaso Antonio Larrañaga, son fuente de análisis, desde

Queremos agradecer especialmente a Juan Justino da Rosa por su ayuda y su generosidad de siempre. El apoyo logístico, desde la Academia Nacional de Letras del Uruguay, de Yamila Montenegro, Raúl Scavarelli y Jimena Hernández facilitó enormemente la realización de esta investigación. A ellos, también, nuestro agradecimiento.

${ }^{2}$ En la actualidad, y a partir de la vigésimo tercera edición de este diccionario, de 2014, se empezó a promover el uso de la sigla DLE para referirse al Diccionario de la lengua española. Sin embargo, en este artículo emplearemos DRAE por el hecho de que los seis trabajos analizados fueron escritos cuando aún era común esta última sigla, y preferimos no alternar entre las dos. 


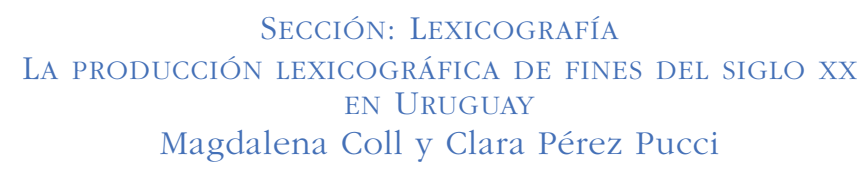

una perspectiva léxico-lexicográfica, para Chans y Urse (2011 y 2012), Almirón y Ochoviet (2011 y 2012) y Coll (2012). Otro mojón fundamental en la historia lexicográfica de esta región es el Vocabulario rioplatense razonado de Daniel Granada, escrito en 1889, que recibió la atención de Kühl de Mones (1986 y 1998), Kornfeld y Kugel (1999), Barcia (2004), López (2017) y, desde una perspectiva glotopolítica, la de Lauria (2010 y 2012). Por su parte, Coll (2012) compara dicho vocabulario con el glosario que Eduardo Acevedo Díaz adjunta a su novela Nativa en 1890. Habrá que incluir también los trabajos de Pereira Rodríguez (1961), Rosell (1978), Elizaincín (2006), Fernández Guerra (2012), Da Rosa y Lucián (2016) y Montenegro (2019), que abordan el El lenguaje del Río de la Plata, obra prácticamente inédita iniciada por Wáshington P. Bermúdez a finales del siglo XIX. Además, habrá que abarcar los diversos trabajos que se ocupan de los varios glosarios que acompañan obras literarias y escritos ensayísticos de fines del siglo XIX, como es el caso de Cabakian (2012), Coll y Montenegro (2012), Coll (2013a, 2013b, 2015 y 2017), entre muchos otros.

Los productos lexicográficos sobre el español del Uruguay escritos en el siglo xx han recibido menos atención, y esta ha sido dispar. Solo por mencionar algunos, diremos que los glosarios de africanismos, escritos por Pereda Valdés (1965) y Laguarda Trías (1969), son el foco del trabajo de Álvarez y Coll (2019) y que la producción lexicográfica de Berro García es abordada en Coll (2018), entre otros. La obra de Guarnieri fue analizada en una tesis de maestría (Soca, 2017) y el Nuevo diccionario de americanismos: Nuevo diccionario de uruguayismos, de Kühl de Mones (1993), ha sido comprendido en varios trabajos que analizan esta obra como parte del proyecto mayor de Günther Haensch y Reinhold Werner (cfr., entre otros, Zimmermann, 2003, y Fajardo Aguirre, 2010).

Una de las razones de la dispar e insuficiente atención metalexicográfica sobre este periodo de la historia lexicográfica del Uruguay está vinculada a la carencia de datos sobre la propia existencia de esa producción. Sin embargo, 


\section{SECCIÓN: LEXICOGRAFÍA \\ LA PRODUCCIÓN LEXICOGRÁFICA DE FINES DEL SIGLO XX EN URUGUAY \\ Magdalena Coll y Clara Pérez Pucci}

a partir de la creación de la base de datos "Daniel Granada" de 500 registros de glosarios, vocabularios y diccionarios escritos en Uruguay o sobre Uruguay, hemos podido tener una mirada global sobre los productos lexicográficos escritos desde fines del siglo XIX. Con este recurso, pudimos tener una perspectiva más amplia del panorama del siglo xx en esta materia, lo que es un paso fundamental hacia la construcción de una historia de la metalexicografía en Uruguay. Seleccionamos, en esta oportunidad, de los datos arrojados por la base, las obras de la segunda mitad del siglo Xx que aun no han sido tratadas por la incipiente actividad metalexicográfica sobre el español del Uruguay o lo han sido de manera tangencial. Así, hemos preferido, en esta oportunidad, concentrarnos en productos lexicográficos poco conocidos, prácticamente no analizados previamente, inéditos en su mayoría o de circulación pequeña o local. Se trata de seis obras, cuyo detalle presentaremos más adelante, que fueron escritas entre 1963 y 1993, lo que nos permite identificar, en definitiva, un periodo de la lexicografía uruguaya que ha recibido muy poca atención de los especialistas.

\section{METODOLOGÍA Y CONFECCIÓN DEL CORPUS}

Nuestro corpus está conformado por obras lexicográficas, escritas en un lapso de treinta años, que se caracterizan, como ya hemos dicho, por tener algún tipo de vinculación con la actividad de la ANL y por reunir un léxico diferencial con respecto a la nomenclatura del DRAE —este será el eje de nuestro análisis-. Destacamos además que este corpus reúne obras que han sido poco o nada exploradas desde una perspectiva metalexicográfica, como ya hemos dicho.

\footnotetext{
${ }^{3}$ Dicha base, financiada por la Comisión Científica de la Universidad de la República, Uruguay, lleva el nombre de "Daniel Granada" en honor al pionero de la lexicografía en Uruguay, y funciona con el software libre de gestión de bibliotecas, PMB. De este modo, constituye la primera compilación sistemática y exhaustiva que se realiza de diccionarios en Uruguay. Reúne diccionarios, glosarios y tesauros publicados autónomamente o anexos a una obra mayor, éditos e inéditos, que tienen sus extremos cronológicos en 1889 y 2020. Se puede consultar en http://basedanielgranada.fic.edu.uy/
} 


\section{SECCIÓN: LEXICOGRAFÍA \\ LA PRODUCCIÓN LEXICOGRÁFICA DE FINES DEL SIGLO XX EN URUGUAY \\ Magdalena Coll y Clara Pérez Pucci}

Las obras en cuestión son: el Vocabulario documentado en la producción literaria de escritores uruguayos (VDPLEU), escrito por César Argüello en 1963; el Diccionario uruguayo documentado (DUD), cuyas autoras son Celia Mieres, Élida Miranda, Eugenia Beinstein de Alberti y Mercedes Rovira de Berro (1966); la obra de Adolfo Berro García, "Vocabulario de uruguayismos" ( $V U)$, publicada en Chile en 1967; el Diccionario documentado de voces uruguayas en Amorim, Espínola, Mas de Ayala, Porta (DDVUAEMP), publicado en 1971 por las mismas cuatro autoras que habían publicado el diccionario de 1966; La vestimenta del Uruguay en el siglo XX. Glosario (VI), elaborado en 1985 —aunque inédito- por Lilián Alba, Carlos Jones y Justino da Rosa, y el trabajo de Rodolfo Tálice, datado en 1993, titulado Cien vocablos biológicos o médicos imperfectamente definidos en la última edición del DRAE (1992) (CVBMIDUED).

Estas obras fueron escritas, como dijimos, entre 1963 y 1993 en Montevideo, aunque el $V U$ fue publicado en Chile. Todas ellas tienen algún tipo de vínculo con la ANL, a partir de diferentes convocatorias o de diferentes preocupaciones personales de académicos, futuros académicos o investigadores de la ANL, como veremos más adelante. Algunas están enfocadas al lenguaje de especialidad (el de la medicina, en el caso del inédito CVBMIDUED, y el de la vestimenta, en el caso del VI). Otras son parte de la tradición de la lexicografía documentada, como se verá en breve. Tres de ellas son inéditas, dos se formalizaron como diccionarios independientes (en 1966 y 1971) y una tiene la estructura de un vocabulario, pero apareció como capítulo de un libro en 1967.

Las dos primeras obras surgen en el marco de la creación de un premio de la ANL, en 1960, sobre el tema "Contribución al Diccionario Histórico de la Lengua Española . Vocabulario documentado en la producción literaria de escritores uruguayos y referido a vocablos, frases, modismos y refranes

\footnotetext{
${ }^{4}$ En las bases se hace referencia al Diccionario histórico de la lengua española. También en el prólogo del DUD y en el VDPLEU. Más allá del adjetivo "histórico" que aparece en esas ocasiones, el diccionario académico que se contrasta con el español del Uruguay es el DRAE, de 1956.
} 


\section{SECCIÓN: LEXICOGRAFÍA \\ LA PRODUCCIÓN LEXICOGRÁFICA DE FINES DEL SIGLO XX EN URUGUAY \\ Magdalena Coll y Clara Pérez Pucci}

no incluidos en el Diccionario de la Lengua Española, Edición 18. ${ }^{a}$, Madrid, 1956, o incorporados en él con otra acepción"s. La obra elegida para ser publicada fue la de cuatro mujeres uruguayas: Mieres, Miranda, Alberti y Berro. Así surgió el DUD (1966).

Fue incorporada a nuestro corpus otra obra que se presentó al concurso, aunque no fue premiada. Es el único trabajo relativo al concurso, además del DUD, que se conserva en el archivo de la ANL. Se trata de una obra que tiene valor como testimonio de la lexicografía de la época y, como tal, se incluyó en nuestro análisis. Es un trabajo inédito escrito por Argüello en 1963, que en su título recoge el nombre del concurso: Vocabulario documentado en la producción literaria de escritores uruguayos y referido a vocablos, frases, modismos y refranes no incluidos en el Diccionario de la Lengua Española. 18 ed. Madrid, 1956 (VDPLEU).

Las autoras de la obra premiada en el concurso escribieron otra, en 1971, basada en cuatro escritores uruguayos: el Diccionario documentado de voces uruguayas en Amorim, Espinola, Mas de Ayala, Porta (DDVUAEMP). Si bien fue escrito por las mismas cuatro autoras que el diccionario de 1966, aparece Alberti como primera autora ${ }^{6}$. Es, en términos metodológicos, una continuación del primero, pero, en esta oportunidad, el foco está puesto en voces que se recogen específicamente en las obras de los autores mencionados en el título del diccionario.

Antes del DDVUAEMP (1971), Berro García publica un conjunto de uruguayismos - "Uruguayismos del habla común" - que conforman un capítulo de

\footnotetext{
${ }^{5}$ Las bases del premio, como se señala en el acta de la ANL del 8 de julio de 1960, establecen que este sería adjudicado cada tres años. Se podrían presentar ciudadanos uruguayos naturales o legales, que entregarían un escrito sobre el tema que la propia Academia determinara para cada edición. Se nombrarían tres ganadores, cuyos trabajos serían publicados por la ANL en un plazo de dos años. No tenemos conocimiento de que se hayan presentado otras obras, más allá de las dos que aquí analizamos.

${ }^{6}$ Estas cuatro autoras también desarrollaron investigación en metalexicografía. Véase, por ejemplo, el artículo titulado "Comentarios a la última edición del Diccionario de la Real Academia Española (1970)", que fue publicado por las autoras en el Boletín de la Academia Nacional de Letras en el año 1973.
} 


\section{SECCIÓN: LEXICOGRAFÍA \\ LA PRODUCCIÓN LEXICOGRÁFICA DE FINES DEL SIGLO XX EN URUGUAY \\ Magdalena Coll y Clara Pérez Pucci}

un libro, editado por Carrillo Herrera, en honor al filólogo chileno Rodolfo Oroz. Es la única obra analizada que fue publicada fuera del Uruguay.

El trabajo inédito de Alba, Jones y Da Rosa, elaborado en 1985, versa sobre el léxico de la indumentaria y nace del premio Conde de Cartagena, impulsado por la RAE en $1982^{7}$. Se titula La vestimenta del Uruguay en el siglo XX. Glosario (VI) y se describe como una recopilación de voces, vinculadas a la indumentaria, que no figuran en la edición XIX del diccionario académico ${ }^{8}$.

Por último, contamos con el trabajo del académico Tálice, datado en 1993. Es inédito, se focaliza en el léxico de la biología o la medicina y lleva por título Cien vocablos biológicos o médicos imperfectamente definidos en la última edición del DRAE (1992) (CVBMIDUED).

En el periodo que nos ocupa, surgieron otras obras que no integran nuestro corpus. La ANL (1980) publicó una selección de paremias usadas en Uruguay pero fue excluida de nuestro corpus porque nos concentramos en compilaciones de voces, excluyendo las de refranes o proverbios. El diccionario de Guarnieri (1979), aunque fue escrito en la época que nos ocupa, no fue incluido porque no es una obra diferencial (cfr. Soca, 2017). Por otra parte, hemos decidido cerrar el extremo cronológico en 1993, fecha en la cual se publicó el Diccionario de uruguayismos de Kühl de Mones (1993), que queda fuera del análisis que aquí nos ocupa. Esta decisión fue tomada en el entendido de que en esa fecha, y a partir de la mencionada obra, la lexicografía uruguaya entra en una nueva etapa, de carácter moderno y científico. La obra de Kühl de Mones es un diccionario muy diferente a los que le preceden, ya sea por su carácter internacional, por los presupuestos teóricos que maneja, por su metodología contrastiva, etcétera. Se distancia, así, del tipo de lexicografía que se estaba haciendo en Uruguay hasta ese momento. Por otra parte, generó reflexiones y análisis metalexicográficos propios, como ya hemos mencionado en la introducción de este trabajo.

\footnotetext{
${ }^{7}$ No encontramos información ampliatoria en el Boletín de la RAE del 1983.

${ }^{8}$ Este material se conserva en el archivo de la ANL: se trata de una copia mecanografiada, que se encuentra intervenida con marcas en lapicera.
} 


\section{SECCIÓN: LEXICOGRAFÍA \\ LA PRODUCCIÓN LEXICOGRÁFICA DE FINES DEL SIGLO XX EN URUGUAY \\ Magdalena Coll y Clara Pérez Pucci}

Las obras elegidas, entonces, fueron analizadas, primero, teniendo en cuenta el contenido de sus prólogos y paratextos, en caso de haberlos, y, luego, atendiendo a la nomenclatura y los artículos que la conforman. En todos los casos, se prestó particular atención al contexto de producción, en el entendido de que algunas fueron hechas "a pedido", como veremos más adelante, ya que nacen como parte de la agenda de la RAE o de la ANL.

\section{ANÁLISIS DE LAS OBRAS}

La "lexicografía poscolonial" (Fajardo Aguirre, 2010) americana se desarrolló a lo largo y ancho del continente con fines y motivaciones diferentes dependiendo de la postura política del autor, del momento histórico, de las circunstancias geolingüísticas y del contexto sociocultural de cada época y región. Se destaca, a los efectos de esta investigación, la vertiente que tiene una historia fuertemente ligada al DRAE, en la medida en que se propone, por lo menos en sus versiones más clásicas, complementar al diccionario académico. Es en esta tradición en la que se inscribe la mayor parte de la lexicografía escrita en Uruguay en los siglos XIX y XX.

En el Río de la Plata, el Vocabulario rioplatense razonado de Granada (1889) y el Lenguaje del Río de la Plata de Bermúdez y Bermúdez (18851947, inédito) surgen "por la incompletud e insuficiencia del DRAE en relación al léxico americano, más precisamente, al léxico de la región rioplatense" (López, 2017). Desde una posición prohispanista la primera, antihispanista la segunda, los dos tienen un carácter diferencial que busca reunir léxico que no figura en el diccionario de la RAE y que pueda ser incluido en este. Son diccionarios construidos de manera tal que los vínculos con el DRAE son explícitos. Las obras lexicográficas que aquí analizamos también tienen un vínculo explícito con el diccionario académico. Incluso en algunos casos el vínculo es tan explícito que se manifiesta en el propio surgimiento de las obras, que nacen "a pedido" de la propia RAE, como veremos más adelante. Pero el contexto es muy diferente al del siglo XIX, ya que, entre otras cosas, las obras del siglo XX 


\section{SECCIÓN: LEXICOGRAFÍA \\ LA PRODUCCIÓN LEXICOGRÁFICA DE FINES DEL SIGLO XX EN URUGUAY \\ Magdalena Coll y Clara Pérez Pucci}

que aquí analizamos nacen en el seno de la ANL, institución que, fundada en 1943, se relaciona regularmente con la RAE desde 1960 y es reconocida, ese mismo año, por la Asociación de Academias de la Lengua Española.

\subsection{VOCABULARIO DOCUMENTADO EN LA PRODUCCIÓN LITERARIA DE ESCRITORES URUGUAYOS}

Como dijimos antes, dos fueron las obras que se presentaron al concurso al que convocó la ANL a principios de la década del 60 del siglo XX. El Vocabulario documentado en la producción literaria de escritores uruguayos (VDPLEU), que data de junio de 1963, tiene en la nota preliminar la siguiente aclaración: "es parte de un trabajo de mayor aliento, en el que venimos laborando, desde años, por mero pasatiempo y sin grandes pretensiones, al margen de nuestras tareas habituales" (s. n.). Es, antes que obra de un especialista, "fruto de la observación en el medio ambiente y del estudio en la literatura vernácula, de un lego en materia lexicológica" (s. n.), en palabras del propio autor. Se propone incluir voces del ámbito rural pero también otras de origen variado, que tienen vigencia en la ciudad, como son aquellas procedentes del lunfardo o de la germanía.

El autor aclara que aquellas voces que figuran en la última edición del diccionario madrileño de la lengua española, al que tilda de "calepino oficial" y "centón", van precedidas de un paréntesis curvo, "cualquiera sea su significado, eso es, igual, similar o distinto al de los consignados por el DRAE" (s.n.), de 1956. Así, en el artículo "arrear" dirá:

(arrear. tr. Llevar violenta y furtivamente ganado ajeno. "Hubo requisa de caballos y algunos fueron arreados al momento ..." J. de Viana. Macachines (VDPLEU, 1963: s. v. arrear).

En batuque, lambeta, renguera no pone esa marca, con lo cual queda implícito que no están en el DRAE consultado.

Luego de una lista con las abreviaturas y las obras y autores en que se basó el trabajo, se presenta la compilación del VDPLEU, que comienza con la voz abacaxí y finaliza en zurubí. Se trata de un extenso trabajo que incluye más de dos mil voces, distribuidas en el cuerpo del texto y en un apéndice. 


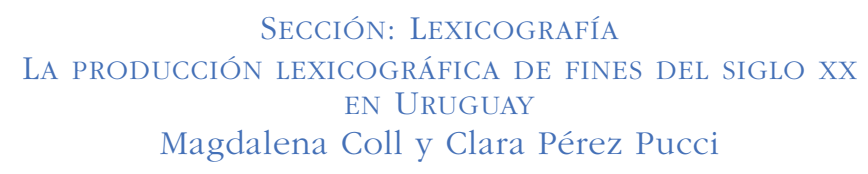

El autor usa marcas gramaticales como $f$., $t r$., y pl., entre otras. También tiene marcas de uso como fam. y fig. o p. us. o vulgar. En algunos casos se presenta una definición sinonímica (por ejemplo, en el artículo pebeta dice chiquilla) y en otros se presentan definiciones analíticas clásicas, que suelen ser breves:

fainá. amb. Pasta delgada hecha con harina de garbanzos, sal y aceite, que se cuece al horno (VDPLEU, 1963: s. v. fainá).

En todos los casos, los artículos tienen ejemplos que documentan estas voces en autores uruguayos, lo que era, como dijimos, requisito del concurso. En el VDPLEU se documentan las voces seleccionadas en prosa, poesía y teatro.

\subsection{DICCIONARIO URUGUAYO DOCUMENTADO}

La otra obra presentada al concurso mencionado, el premiado Diccionario uruguayo documentado (DUD, 1966), busca, según reza el prólogo escrito por las autoras, presentar vocablos que no figuran en el DRAE (también refiere a la edición de 1956), aunque sí se consignan las voces que figuran en el diccionario académico como arcaísmos. Estos eran considerados arcaísmos en el español peninsular, pero estaban vigentes en el español del Uruguay, a entender de las autoras.

Se incluyeron algunos vocablos que constituyen neologismos, pero cuyo uso se reduce a la literatura. Las autoras consideran que "pueden significar un punto de partida en la lengua literaria, aunque no [hayan] podido comprobarlo" (DUD, 1966, p. 7).

El prólogo concluye con un listado de "voces usadas por escritores uruguayos que el diccionario de la Real Academia Española atribuye a otras naciones americanas". Son 114 en total, entre las que se consignan coima (registrada en el El paisano Aguilar, de Enrique Amorim y en El país de la cola de paja, de Mario Benedetti), firulete (en el El paisano Aguilar y El terruño, de Carlos Reyles) y tordo (recogida en Ismael, de Eduardo Acevedo Díaz).

Luego de este listado, comienza el vocabulario propiamente dicho, que va desde a babucha hasta zumbante. En los artículos, se incluyen, en algunos pocos casos, referencias al DRAE y al Diccionario manual e ilustrado de la 


\section{SECCIÓN: LEXICOGRAFÍA \\ LA PRODUCCIÓN LEXICOGRÁFICA DE FINES DEL SIGLO XX EN URUGUAY \\ Magdalena Coll y Clara Pérez Pucci}

lengua española (1950), también de la RAE. En esas oportunidades se remite a un equivalente que aparezca en los diccionarios mencionados y luego se reproducen las definiciones que allí se consignan. Por ejemplo:

desocuparse. $r$. Alumbrar, acep. 10. RAED, ocupada. adj. Dícese de la mujer preñada (DUD, 1966: s.v. desocuparse).

cocotte (voz francesa). f. Prostituta. RAEM. [...] * Cocote. f. Ramera (DUD, 1966: s.v. cocotte).

También se reproducen otras definiciones de otros diccionarios como el Diccionario de americanismos (Malaret, 1946) o el Diccionario ideológico de la lengua española (Casares, 1942).

\subsection{DicCionario DOCUMENTAdo DE VOCES URUGUAYAS EN AMORIM, ESPÍNOLA, MAS DE AYALA, PORTA}

Pocos años después, en 1971, se publicó el Diccionario documentado de voces uruguayas en Amorim, Espínola, Mas de Ayala, Porta (DDVUAEMP), escrito por las mismas cuatro autoras. Se trata de una publicación que retoma el trabajo del $D U D$, como ya dijimos, pero que está focalizada en cuatro escritores uruguayos. Esta obra, que tiene entre sus objetivos reivindicar y dar a conocer el habla del Uruguay, también recoge únicamente voces que no fueron tenidas en cuenta en el DRAE o aquellas que no presentan las marcas referidas al Uruguay. La edición del diccionario de la Academia que tomaron de referencia fue la decimoctava, de 1956. Sin embargo, mientras la obra de estas autoras estaba en proceso editorial, se publicó la decimonovena edición del DRAE, en 1970, en la que se incluían algunas de las voces que recogieron para su obra de $1971^{9}$. Esto ocurrió porque la RAE había tenido en consideración la anterior obra de las autoras (DUD, 1966), además de una presentación que Esther de

\footnotetext{
${ }^{9}$ Esta información se encuentra también en el prólogo de Mil palabras del español del Uruguay (ANL, 1998, pp. 5-7), en el que se explicita que la vinculación de estas autoras al quehacer académico y la presencia en la Secretaría de la ANL de Esther de Cáceres y su viaje a Madrid, en 1968, para integrar la Comisión Permanente de la Asociación de Academias, dieron un impulso a la lexicografía académica uruguaya. La mayor parte de los uruguayismos registrados en el DRAE a partir de su edición decimonona tienen su origen en la actividad de estas mujeres.
} 


\section{SECCIÓN: LEXICOGRAFÍA \\ LA PRODUCCIÓN LEXICOGRÁFICA DE FINES DEL SIGLO XX EN URUGUAY \\ Magdalena Coll y Clara Pérez Pucci}

Cáceres hizo en la Comisión Permanente de Academias de Lengua (Madrid, 1968). Las voces ya incluidas en el DRAE no fueron quitadas de la publicación de 1971 porque las autoras consideraron que presentaban un valioso valor documental —en sus propias palabras_- pero sí fueron señaladas con la frase "Incorporado a D.R.A.E, 70". Tal es el caso de cuadra, gurí, masita ${ }^{10}$.

El prólogo, mucho más extenso que el presentado en la obra de 1966, fue escrito por las propias autoras, y allí se indica que fueron adoptadas las abreviaturas del DRAE. Se explicita que el criterio adoptado es el de recoger palabras, provenientes de textos literarios de escritores uruguayos, que hayan quedado "fuera de la 'norma' española fijada en sus diccionarios (1956 y ahora 1970) [...]. De modo que [...] [dan] a la imprenta alrededor de dos millares de palabras que emplean los uruguayos, que no están en el español general" (DDVUAEMP, 1971, p. 12). Las autoras aclaran que son dos mil en total las voces incluidas —que no se encuentran en el "español general"-, todas ellas documentadas. Aunque no es este el lugar para ahondar en esta discusión, cabe notar que entrecomillan la palabra "norma" al relacionarla con el "español general".

En el prólogo, además, se mencionan algunos extranjerismos crudos y se señala que algunos de ellos fueron incluidos en el diccionario de la RAE en su forma adaptada al español, cuando en Uruguay aún se usaba su forma extranjera. Uno de ellos es maquette/maqueta, cuyo artículo figura de la siguiente forma:

MAQUETTE: (Voz francesa) f. Maqueta.

Montevideo y su cerro, 65:

Los estudiantes de Arquitectura, preparando también su viaje, rifaban un chalet, situado en una localidad llamada Carrasco, y la maquette de ese chalet, de tres metros de ancho, se atravesaba al paso de los peatones (DDVUAEMP, 1973: s.v. maquette).

\footnotetext{
${ }^{10}$ Curiosamente, las voces que las autoras ejemplifican en el prólogo no figuran en el cuerpo de ese mismo diccionario.
} 


\section{SECCIÓN: LEXICOGRAFÍA \\ LA PRODUCCIÓN LEXICOGRÁFICA DE FINES DEL SIGLO XX EN URUGUAY \\ Magdalena Coll y Clara Pérez Pucci}

Las fuentes de las que fueron recogidas las voces del diccionario pertenecen a seis obras de los cuatro escritores que figuran en su título, ya que uno de los objetivos del trabajo es plasmar el uso de los vocablos a partir de cómo fueron usados por los autores estudiados. En este sentido, el lemario de esta obra es cerrado y está acotado por la producción de los escritores elegidos. Estos fueron seleccionados en el entendido de que la lengua que utilizan es representativa del habla uruguaya en casi toda su extensión geográfica y en su diversidad de registros.

Se señalan particularmente los ejemplares de flora y fauna que en el diccionario de la RAE se definen haciendo alusión a especímenes distintos que aquellos a los que refiere el mismo nombre en América y particularmente en Uruguay. Al respecto, las autoras indican que "sería conveniente que en la próxima edición el DRAE precisara esta nominación que abarca amplio espacio en América" (DDVUAEMP, 1971, p. 18). Sin embargo, no parece que esto haya sido tenido en cuenta en la siguiente edición del DRAE. Algunos ejemplos que entran en esta categoría son la comadreja, el cuervo y el burucuyá.

Luego, se describe en el prólogo el proceso de incorporación de una voz al diccionario de las autoras. En primer lugar, se la identifica en la obra literaria y se consultan diccionarios de la RAE (como el de 1956 y el Diccionario manual e ilustrado de la lengua española, de 1950) y materiales como el Boletín de la Asociación de Academias de la Lengua Española. Si la voz no se encuentra en estas fuentes, es probable —entienden las autoras- que sea un americanismo. Si se encuentra, cabe la posibilidad de que se haya recogido con un significado distinto al que se usa en Uruguay. El siguiente paso es determinar si la voz es únicamente uruguaya o si se utiliza en otras partes de América, pero las autoras entienden que caracterizar a una voz como uruguayismo supone "una investigación rigurosa y pormenorizada" (DDVUAEMP, 1971, p. 23), que no siempre es posible realizar.

Para dar con el campo semántico de la voz, se recurre a diccionarios americanos especializados, vocabularios y monografías, a obras literarias y a 


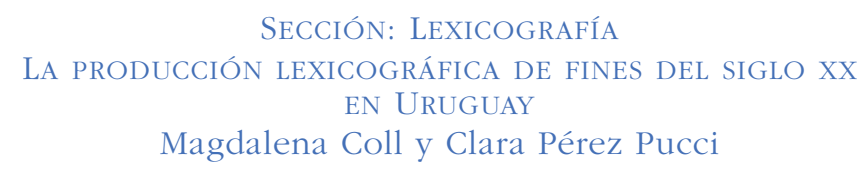

la propia competencia de hablante de las autoras. Luego, se clasifica la voz según su clase de palabra y se señala su ámbito habitual de uso. Es una de las pocas obras que aquí analizamos en la que se explicita en detalle la metodología del trabajo lexicográfico.

Las autoras aclaran cuando las voces están en el DRAE pero sin la marca $U r$. Tal es el caso de la voz acollarar, en la que se especifica que el diccionario de la Academia la registra para Argentina pero no para Uruguay, aunque tiene en ambos países el mismo significado y uso. Igual situación se describe para achura, aguatero o ahijuna. Las autoras también aclaran cuando la voz aparece en el DRAE pero con otras acepciones que no son las que se usan en Uruguay, como en alzaprima. Además, comentan las voces que aparecen con una forma similar en el DRAE, como a raja-cincha que se registra como a revienta cinchas. Se detienen asimismo en aspectos de lexicografía práctica al afirmar que hay voces que aparecen en el diccionario de la RAE pero lematizadas de otra manera: andar ido aparece en el DRAE en el artículo ir, estar ido, con la significación "estar distraído". Las autoras lo consignan, sin embargo, por la $a$, en el artículo andar ido.

La microestructura, tanto en la obra de las autoras de 1966 como en la de 1971, incluye marca gramatical y una definición sencilla. Cada artículo se acompaña — y esta es la esencia de la obra - de ejemplos de uso en las obras de los autores seleccionados, como ya dijimos. Por ejemplo, en el artículo rambla, de la edición de 1971, después de la marca gramatical (f.) y la definición ("avenida costanera") se transcribe un ejemplo tomado de la novela Montevideo y su cerro, de Mas de Ayala: "Por la escollera —fina estocada de piedra que la calle Sarandí lanza al mar - retornan con sus cañas al hombro los pescadores. Parejas de enamorados, como esculturas de Eros, van deorando la Rambla" (DDVUAEMP, 1973: s. v. rambla).

Las obras de estas cuatro autoras tienen dos diferencias entre sí. Por un lado, la primera (de 1966) se basa en varios autores uruguayos, mientras que la segunda (que es de 1971) se concentra en cuatro, como ya dijimos. 


\section{SECCIÓN: LEXICOGRAFÍA \\ LA PRODUCCIÓN LEXICOGRÁFICA DE FINES DEL SIGLO XX EN URUGUAY \\ Magdalena Coll y Clara Pérez Pucci}

Por otro lado, la obra de 1971 es ilustrada: varios artículos tienen un dibujo, hecho por Domingo Ferreira, que además de instruir sobre el significado en cuestión le dan a la edición una estética propia.

\subsection{VOCABULARIO DE URUGUAYTSMOS}

Previo a este segundo diccionario de las cuatro autoras, que analizamos junto a su primera obra, Berro García había publicado en 1967 un vocabulario que llevó por título "Vocabulario de uruguayismos"11 ( $V U$ ). Es un adelanto de una obra mayor, el Vocabulario del habla popular uruguaya, que hubiera abarcado unas diez mil voces, pero que no llegó a publicarse:

El pequeño Vocabulario de Uruguayismos que va a continuación es solo una parte mínima, hemos reunido 10.000 voces, acepciones nuevas y giros y frases usadas en el Uruguay y que se hallan ausentes del diccionario de Madrid.

Este Vocabulario de Uruguayismos, que abarcará dos volúmenes de 400 páginas cada uno, se halla en trance de impresión, buscándose la forma de financiar la obra por intermedio del Ministerio de Instrucción Pública Nacional ${ }^{12}$ ( $V U, 1967$, p. 53).

Este trabajo fue realizado por un equipo de docentes, coordinado por el mismo Berro García, que "recorren en giras el territorio nacional y realizan, desde hace ya tres años, el levantamiento de la Encuesta Idiomática Nacional destinada a recoger, seleccionar, fichar y registrar las voces, giros, frases hechas y figuradas que a centenares se obtienen en los trabajos de campo realizados por los referidos cuestores [sic], a los que se agregan los colaboradores, distribuidos por toda la República" ( $V U, 1967$, p. 53).

El objetivo era contribuir a la elaboración de los atlas lingüísticos de toda América, en coordinación con las Juntas de Investigación del Habla Popular Hispanoamericana. El $V U$ se enmarca, entonces, en un proyecto iberoamericano, y, en ese sentido, es un trabajo particularmente distinto del resto de los que aquí analizamos. Sin embargo, por muchos factores que no

\footnotetext{
${ }^{11}$ Nos basamos aquí en el análisis que hiciera Coll (2018) sobre la obra lexicográfica de Berro García.

${ }^{12}$ No hay documentación de que se haya conseguido esa financiación ni se conservan, hasta donde llega nuestro conocimiento, los materiales y fichas sobre esas voces.
} 


\section{SECCIÓN: LEXICOGRAFÍA \\ LA PRODUCCIÓN LEXICOGRÁFICA DE FINES DEL SIGLO XX \\ EN URUGUAY \\ Magdalena Coll y Clara Pérez Pucci}

desarrollaremos aquí, el proyecto internacional que promovió Berro García no llegó a buen puerto. Y este vocabulario, última publicación de su autoría, no hace justicia a la propuesta original ${ }^{13}$.

El autor aclara que la mayoría de las voces recogidas proviene de "las palabras y raíces ya adaptadas por el español, pero cuyo significado ha variado, a veces radicalmente. Son nuevas y nuevas acepciones de los vocablos castellanos" mientras que otras voces "provienen de las raíces existentes en la lengua, pero que se han modificado en su valor y significado, por ágiles medios de que disponen nuestras lenguas neoromances, por la derivación y la composición, empleando los centenares de prefijos y sufijos que se hallan a nuestra disposición" ( $V U, 1967$, p. 54).

\section{Y continúa:}

Una serie, hoy importante, toma procedencia mediante el préstamo de voces y giros foráneos a nuestra lengua, ya sea de las lenguas extranjeras de mayor influencia en las comunicaciones universales, como son el inglés y el francés, el italiano y el portugués, como asimismo de los idiomas autóctonos de América, particularmente para el Uruguay el guaraní-tupí, el quechua y el mapuche.

Por lo demás, en materia de giros, frases hechas, refranes, símiles, frases figuradas, etc., existe una formidable gama de expresiones que por miles y miles enriquecen, adornan, y euforizan nuestra habla común, singularmente por los habitantes de nuestra campaña, que, como el Sancho Panza de la inmortal obra, crean, endilgan, robustecen y dan emotividad formidable al habla campesina y vulgar ( $V U, 1967$, p. 54).

\footnotetext{
${ }^{13}$ La reseña de este trabajo que hace De Granda, en 1968, dista de ser favorable: "Nuestra sensación de extrañeza ante este trabajo [el de Berro García] quizá provenga (así queremos suponerlo) de la defectuosa exposición que el autor hace de la finalidad del mismo. Pues si, como parece desprenderse de los párrafos introductorios e incluso en el título, Berro se propuso presentar un muestrario de voces limitadas extensivamente al Uruguay, no comprendemos la inclusión en el mismo de expresiones de uso general hispánico como ancestral, apartamento, a diario, avatar, bagaje, banal, bebé, cabaña, camionero, carnet, casino, constatar, etc. Ahora bien, si lo que el autor se propuso fue, simplemente, recoger un breve muestrario del léxico común uruguayo (pero no limitado al Uruguay), nuestra extrañeza desaparece, aunque no veamos la utilidad de un trabajo semejante, solo comprensible enfocado exhaustivamente respecto al habla de un territorio o localidad determinados" (p. 96). Continúa en la reseña criticando algunos errores tipográficos que aparecen en la publicación (que califica De Granda de "extraños detalles"). Como no hemos encontrado en el original los errores mencionados, es bastante probable que hayan surgido en el proceso de edición.
} 


\section{SECCIÓN: LEXICOGRAFÍA \\ LA PRODUCCIÓN LEXICOGRÁFICA DE FINES DEL SIGLO XX EN URUGUAY \\ Magdalena Coll y Clara Pérez Pucci}

Son unos 260 artículos, que van de abatarse a Yaro. Tienen algunas marcas gramaticales como refl. adj., tr., m., entre otras. El autor aclara cuando la RAE le da otra acepción a la voz, como en "abatarse, refl. Asustarse, amilanarse, entregarse moralmente. Perder el ánimo" ( $U$, 1967: s. v. abatarse), que aparece en el DRAE con otro significado. También señala otros aspectos vinculados al diccionario académico. Por ejemplo, en el artículo agilizar, afirma: "La Academia Española trae las dos grafías [...] agilizar/agilitar", pero expresa que la primera forma es la "preferible por ser más eufónica a la de agilitar" ( $V U, 1967:$ s. v. agilizar).

\subsection{VOCES DE LA INDUMENTARIA}

Este trabajo inédito de Alba, Jones y Da Rosa (VI) recoge "voces de uso actual que no figuran en el Diccionario académico (edición XIX)" (p. IV), es decir, el $D R A E-70$, en un área concreta del lenguaje de especialidad como es el de la vestimenta. Además, se deja constancia de aquellas voces que fueron incorporadas en la vigésima edición, publicada poco después de escrito este vocabulario, y se marcan también, con un asterisco, aquellas voces que figuran en el diccionario de la Real Academia, pero con otro significado.

Las voces son de uso en Uruguay, pero también se recogen otras registradas en Argentina (Buenos Aires) y España (Madrid y Barcelona). Fueron tomadas de publicaciones sobre la vestimenta, no solamente de diccionarios editados en esas ciudades.

En la bibliografía fundamental hay otros dos diccionarios españoles además del DRAE: Diccionario de uso del español, de María Moliner, y Diccionario general ilustrado de la lengua española, de Vox. En este sentido, no se le adjudica a la Real Academia la completa hegemonía respecto del registro de uso de la lengua en España.

Cada artículo tiene su etimología, su significado, marcas geográficas de uso y varios ejemplos. También se ponen las siglas de las obras lexicográficas en que aparece la palabra en cuestión. Tal es el caso de 


\section{SECCIÓN: LEXICOGRAFÍA \\ LA PRODUCCIÓN LEXICOGRÁFICA DE FINES DEL SIGLO XX EN URUGUAY \\ Magdalena Coll y Clara Pérez Pucci}

casco. m.

Sombrero femenino semiesférico y sin ala.

Reg. en: VOX.

"(...) "Allo Paris" es un tocado para la noche. Un casquito con dos rodetes a los costados cubiertos de tul (...)"

MU14, Mvdeo., 1/5/47, p. 59, "Los sombreros de París".

"(...) Y aquí y allá el ya famoso casco estilo Garbo aparece (...)"

SFD ${ }^{15}$, Mvdeo., 28/8/81, p. 7 (VI, 1985: s. v. casco).

Se trata, sin duda, de un minucioso trabajo de recopilación diferencial, que, desde una autoría colectiva, se focaliza en un área específica de la lengua: el lenguaje de la vestimenta.

\subsection{CIEN VOCABLOS BIOLÓGICOS O MÉDICOS IMPERFECTAMENTE DEFINIDOS EN LA ÚLTIMA EDICIÓN DEL DRAE (1992)}

La última obra que presentaremos fue escrita por el académico Tálice, Cien vocablos biológicos o médicos imperfectamente definidos en la última edición del DRAE (1992) (CVBMIDUED). Data de 1993, pero permanece inédita. Comienza con una justificación en la que el autor explica que va a recopilar "imperfecciones" del DRAE, "peccata minuta", aunque algunas podrían considerarse, según él mismo, "peccata majore". Incluye definiciones incompletas, imprecisiones, definiciones defectuosas y anticuadas que encuentra en el DRAE.

El autor hace una "revisión de los vocablos médico-biológicos del DRAE", inspirado "en algunos conceptos generales que no parecen orientar a los especialistas de la Academia" (CVBMIDUED, 1993, p. 6). Enumera cuatro puntos que considera que en el diccionario de la Academia no se resuelven satisfactoriamente: la inclusión de demasiados detalles anatómicos externos de los insectos vectores de enfermedades, la falta de énfasis en el registro de infecciones que transmiten estos insectos, la falta de datos sobre la distribución geográfica de los insectos y la ausencia de información sobre el grado de curabilidad de las enfermedades presentadas (CVBMIDUED, 1993).

\footnotetext{
${ }^{14}$ Revista Mundo Uruguayo (Montevideo, 1919-1967).

15 "Suplemento familiar" de El Día (Montevideo, 1970-1983).
} 


\section{SECCIÓN: LEXICOGRAFÍA \\ LA PRODUCCIÓN LEXICOGRÁFICA DE FINES DEL SIGLO XX EN URUGUAY \\ Magdalena Coll y Clara Pérez Pucci}

La nomenclatura, entonces, se organiza en función de la intención de revisar vocablos médico-biológicos del DRAE. En los aproximadamente cien artículos de este diccionario, se encuentran muchos vinculados a la biología en general o la medicina en particular, como es el caso de dengue, espiroqueta y pediculosis. También se incluyen muchos artículos de animales, como cigüeña o comadreja, que estarían más vinculados con la zoología en particular, y también voces de la flora, como espinillo y frutilla. Por otra parte, una gran cantidad de lemas no provienen de ninguna de estas áreas y su pertenencia a este trabajo es difícil de sostener, como clarividencia, simposio o golf. Este hecho pone de manifiesto el carácter amateur de la lexicografía del autor de CVBMIDUED: si bien su trabajo de corrección del diccionario de la Real Academia es muy meticuloso, carece de sistematicidad metodológica a la hora de la delimitación del lemario con el que trabaja.

El formato de CVBMIDUED es el siguiente: se presenta la definición que da el $D R A E$ de una palabra y, debajo, la crítica del autor. Luego se brinda una propuesta de sustitución de la definición. Se la redacta de nuevo íntegramente, respetando en todos los casos la etimología de la primera versión. Es explícitamente una obra abocada a corregir el DRAE y es la única de las obras aquí analizadas que propone definiciones sustitutivas a las académicas, previa caracterización de los errores o imprecisiones de la definición del DRAE. Tal es el caso del artículo para instinto:

INSTINTO. (Del lat. instinctus.) m. Conjunto de pautas de reacción que, en los animales, contribuyen a la conservación de la vida del individuo y de la especie. Instinto reproductor.

Crítica. Imperfecta. Según la Etología el sentido moderno del vocablo sustituido por el de Comportamiento instintivo es aquel cuyo origen es la herencia biológica, a través de los genes, que tiene siempre carácter adaptativo.

Proposición. Instinto. (Del lat. instinctus.) m. Modernamente conjunto de pautas comportamentales adaptativas originadas por la herencia biológica a través de los genes. Lo estudia la Etología (CVBMIDUED, 1993: s. v. instinto).

Las correcciones que hace el autor en los artículos se pueden agrupar en tres grandes clases: corrección de la distribución geográfica (en general, para 


\section{SECCIÓN: LEXICOGRAFÍA \\ LA PRODUCCIÓN LEXICOGRÁFICA DE FINES DEL SIGLO XX EN URUGUAY \\ Magdalena Coll y Clara Pérez Pucci}

precisar que un vocablo se utiliza en Uruguay y Argentina), corrección de imprecisiones en algunas partes de la definición y ampliación de la definición a partir de la planteada por la RAE. Se trata de una clasificación original y útil.

La revisión de los artículos, que van desde ácido salicílico hasta zarigüeya, es muy minuciosa, al punto de que en algunos casos las observaciones pueden parecer nimiedades para el lector común. Sin embargo, Tálice, en la medida en que entiende que muchas de las definiciones de la RAE son "perfeccionables", propone modificaciones aunque supongan solo el cambio de una o dos palabras.

En las ediciones posteriores del DRAE no figura ninguna de las propuestas del autor. Teniendo en cuenta el carácter inédito de este escrito, es altamente probable que los académicos de la RAE no hayan sabido de su existencia.

\subsection{Síntesis de los datos analizados}

Del análisis realizado surgen algunos puntos de comparación entre las obras de nuestro corpus. Con respecto a los paratextos, solo algunas contienen un prólogo, que suele ser breve, a excepción del DDVUAEMP (1971). Solo el DUD (1966) contiene un prólogo y una "advertencia", que fue escrita por el académico Ariosto D. González. Los prólogos, si los hay, están escritos por los mismos autores de la obra. Se recurre, solo en algunos casos, a la presentación de un listado de las voces consignadas.

La extensión de las obras abarca desde las cien voces (como es el caso del CVBMIDUED) hasta las dos mil (cfr. DDVUAEMP, 1971). Se recogen extranjerismos, voces patrimoniales y excepcionalmente neologismos, siempre con un criterio diferencial con respecto al DRAE. La microestructura es sencilla. Es excepcional la aparición de etimologías. Se manejan, principalmente, las indicaciones gramaticales de clases de palabras y marcas de uso como vulgar o fam., aunque también se pueden encontrar marcas geográficas como Arg. Las abreviaturas, cuyo listado se incluye al inicio de las obras, responden al uso tradicional en lexicografía y coinciden, la mayoría de las veces, con las usadas en el DRAE. Las definiciones son sencillas; remiten a equivalentes o son de 


\section{SECCIÓN: LEXICOGRAFÍA \\ LA PRODUCCIÓN LEXICOGRÁFICA DE FINES DEL SIGLO XX EN URUGUAY \\ Magdalena Coll y Clara Pérez Pucci}

tipo analítico, pero siempre breves. Los ejemplos que se incluyen funcionan como documentación de uso de escritores de la época. En el caso del vocabulario sobre la vestimenta, se consignan ejemplos de diarios y periódicos de la época. No hay ejemplos creados por los autores.

De los datos analizados surgen también tendencias, inclinaciones y preferencias que hacen a la lexicografía de fines del siglo pasado en Uruguay. Serán presentadas a continuación.

\section{CONSIDERACIONES FINALES}

Como ya hemos dicho, nuestro corpus está conformado por obras lexicográficas, escritas en un lapso de treinta años, que se caracterizan por estar vinculadas en distinta medida con la ANL y tener un carácter diferencial con respecto al diccionario de la RAE.

De la descripción y análisis de estas obras, que han recibido, hasta ahora, nula o escasa atención por parte de la comunidad académica, surgen algunas tendencias de la lexicografía uruguaya de fines del siglo xx. Parece claro que el concurso promovido por la ANL a principio de la década del 60 dio un impulso a esta producción lexicográfica, un impulso que apunta a ser una contribución al diccionario madrileño. Resultado de esa instancia, contamos hoy con el DUD (1966), que dio lugar a un trabajo posterior (DDVUAEMP, 1971). El VDPLEU, aunque permanece inédito, también nace de esta convocatoria. Por otra parte, la convocatoria de la RAE de 1982 promovió el trabajo sobre el léxico de la vestimenta (VI).

La autoría de la mayoría de los productos lexicográficos que hemos visto es de académicos de la Anl (Mieres, Miranda, Berro García, Tálice) o de investigadores de dicha institución, como es el caso de Jones y Da Rosa, que ingresaron como académicos en fecha posterior a la elaboración de sus trabajos. Pero se trata de emprendimientos de muy distinta índole. Por ejemplo, el trabajo de Tálice (CVBMIDUED) es un aporte personal, pero el vocabulario de Berro García $(V U)$ forma parte de un proyecto de dimensión iberoamericana, 


\section{SECCIÓN: LEXICOGRAFÍA \\ LA PRODUCCIÓN LEXICOGRÁFICA DE FINES DEL SIGLO XX EN URUGUAY \\ Magdalena Coll y Clara Pérez Pucci}

que tenía el apoyo de la ANL. En esto, entre otros motivos, radica la diferencia de estilo y formato entre las obras.

La variable género aporta datos importantes. Se consolida en esta época el trabajo de cuatro mujeres: Mieres, Miranda, Alberti y Berro. Otra mujer, Alba, es una de las autoras del trabajo sobre indumentaria. Así las cosas, las mujeres no parecen marginadas de esta área de conocimiento. Se destaca el hecho, además, de que las mujeres de este periodo trabajan de manera colectiva: no hay obras escritas individualmente por una mujer. Del mismo modo, no hay obras colectivas escritas solo por hombres.

Con respecto a la formación de los autores, muchos de ellos se dedican a la enseñanza del español o la literatura o eran maestros (Berro García, Jones, Berro, Mieres, Miranda, Alberti, Da Rosa, Alba). En esa época no había formación específica en lexicografía en Uruguay — como todavía no la hay- ni en lingüística ${ }^{16}$. Algunos son legos en lexicografía, como es el caso de Tálice, de profesión médico, o el caso de Argüello, que se describe como no especialista. Pero otros, como Mieres, Miranda, Berro, Alberti y Berro García, tienen experiencia o fueron generando experiencia en lexicografía y participaron, de una u otra forma, en la Comisión de Lexicografía de la ANL. Entre ellos, se destaca Berro García, abogado de profesión, que dirigió la Comisión de Lexicografía, tuvo experiencia en trabajo de campo en lexicografía y coordinó un equipo de colaboradores con el cual desarrolló actividades de índole lexicográfica en Uruguay por décadas.

Buena parte de la producción lexicográfica que hemos analizado permanece inédita ( CVBMIDUED, VDPLEU y VI), aunque tienen la intención de ser aportes

\footnotetext{
${ }^{16}$ Apenas con la Escuela de Lexicografía, instituida en Madrid en 2001 por la Real Academia Española y la Asociación de Academias de la Lengua Española (https://www.rae.es/la-institucion/escuela-de-lexicografia-hispanica), se fue consolidando la formación de investigadores uruguayos en lexicografía. Aunque la licenciatura en Letras, de la entonces Facultad de Humanidades y Ciencias, Universidad de la República, Uruguay, ofrecía una especialización en Lingüística, es apenas en la década del 70 del siglo pasado cuando se crea la licenciatura en Lingüística.
} 


\section{SECCIÓN: LEXICOGRAFÍA \\ LA PRODUCCIÓN LEXICOGRÁFICA DE FINES DEL SIGLO XX EN URUGUAY \\ Magdalena Coll y Clara Pérez Pucci}

al diccionario de la Real Academia. Los inéditos no logran serlo, precisamente por su carácter de inédito; no logran tampoco tener impacto en la lexicografía nacional. En cambio, el DUD se destaca por haber generado cambios en el diccionario de Madrid, tal cual se explica en el prólogo del DDVUAEMP (1971) y en el de Mil palabras del español del Uruguay (ANL, 1998) ${ }^{17}$. En este sentido, podemos recordar lo que pasó con las obras decimonónicas de la lexicografía uruguaya: algunas voces del vocabulario de Granada, publicado en 1889, fueron de alguna manera recogidas por el DRAE con el pasar del tiempo. La obra de Bermúdez, que permanece inédita, no fue (re)conocida en Madrid y no se instaló como una obra de referencia en la región, como sí lo hizo Granada.

El carácter diferencial de estas obras se pone de manifiesto de diferentes maneras. En primer lugar, es diferente la edición del DRAE con la cual contrastan: las del concurso de ANL, con la de 1956, que es la decimoctava; la de la indumentaria toma como referencia de contraste la edición decimonovena, que es del año 1970; CVBMIDUED, con la de 1992, que es la vigesimoprimera. En el $V U$ no se explicita ninguna edición, pero es razonable suponer que se trata de la decimoctava, ya que era la última edición en 1967, cuando se publicó su capítulo.

En muchos casos, este contraste es sumamente explícito, incluso en el título de la obra (por ejemplo, el de CVBMIDUED), en otros casos se manifiesta en el prólogo y se recoge en el cuerpo de la obra. Otras veces no aparece información en los paratextos de la obra pero sí en el desarrollo de la microestructura. Por otra parte, algunas obras proponen, a grandes rasgos, que ciertas voces sean incluidas en el DRAE, es decir, apuntan a cambios en el lemario; al menos, ese es el énfasis. El caso de CVBMIDUED es particular porque tiene una clara intención de generar cambios en la microestructura

\footnotetext{
${ }^{17}$ Cabe destacar que el trabajo de Héctor Balsas (1977) está dedicado precisamente a mostrar los uruguayismos que fueron integrados al diccionario académico de 1970. Desconocemos si existe un trabajo exhaustivo y detallado sobre el proceso diacrónico de incorporación de voces del Uruguay al DRAE.
} 


\section{SECCIÓN: LEXICOGRAFÍA \\ LA PRODUCCIÓN LEXICOGRÁFICA DE FINES DEL SIGLO XX EN URUGUAY \\ Magdalena Coll y Clara Pérez Pucci}

del DRAE (1992). A su vez, la manera en que se contrasta con el DRAE no es siempre igual y solo en los casos de CVBMIDUED y el DDVUAEMP se explicita la metodología de contraste. Además, el contraste se fundamenta en registros de época en DUD, DDVUAEMP, VDPLEU y VI. En este sentido, estas obras son documentadas, a diferencia del resto, que no lo son.

Resaltamos el hecho de que las obras que aquí analizamos, al buscar contribuir al DRAE, generan un valor propio de descripción léxico-lexicográfica del español del Uruguay. Generan tradición y aportan al estudio léxico uruguayo, aunque no surgieron necesariamente con ese propósito.

Mientras se escriben o publican las seis obras que hemos visto, también se están elaborando en la época dos obras fundamentales de la lexicografía uruguaya: el Nuevo diccionario de uruguayismos (Kühl de Mones, 1993) y el Diccionario del español del Uruguay, DEU, que se publicará en 2011. Así, es una época, entonces, en la que, en simultáneo a las obras estudiadas, se están gestando dos obras emblemáticas para la lexicografía uruguaya del siglo xx y xxI. Este es el escenario de la lexicografía en la segunda mitad del siglo Xx en el Uruguay: en él conviven, por un lado, una lexicografía que tiene una divulgación muy restringida, que se origina, por lo general, en el ámbito de la ANL y que ha recibido, hasta ahora, muy poca atención de parte de la comunidad académica. Por otro, se van consolidando las dos obras pioneras de la lexicografía uruguaya moderna. En la última de ellas, el DEU, participan activamente varios de los actores que ya venían trabajando en la lexicografía que usamos como corpus en este artículo. Así, analizar las características de las obras que aquí presentamos nos ayuda a ver diferentes eslabones en la historia de la lexicografía —y de la metalexicografía- uruguaya.

\section{BIBLIOGRAFIA}

Academia Nacional de Letras (1980). Selección de paremias. Imprenta Cordón. Academia Nacional de Letras (1998). Mil palabras del español del Uruguay. Banda Oriental. Almirón, S., y Ochoviet, S. (13 de octubre de 2011). El lenguaje de Dámaso Antonio Larrañaga: el caso de leñatero. V Seminario sobre lexicología y lexicografía del español 


\section{SECCIÓN: LEXICOGRAFÍA \\ LA PRODUCCIÓN LEXICOGRÁFICA DE FINES DEL SIGLO XX \\ EN URUGUAY \\ Magdalena Coll y Clara Pérez Pucci}

y del portugués americanos: a 200 años del inicio del proceso independentista del Uruguay, Montevideo, Uruguay. http://www.academiadeletras.gub.uy/innovaportal/ file/102847/1/almiron-ochoviet.pdf

Almirón, S., y Ochoviet, S. (16-17 de octubre de 2012). El lenguaje de Dámaso A. Larrañaga: los diminutivos. VI Seminario sobre lexicología y lexicografía del español y del portugués americanos, Montevideo, Uruguay. http://www.academiadeletras.gub.uy/ innovaportal/file/102862/1/almiron-ochoviet.pdf

Álvarez, L., Coll, M. (2019). Registers of African-derived lexicon in Uruguay: etymologies, demography and semantic change. Zeitschrift für romanische Philologie, 135(1), 223255. https://doi.org/10.1515/zrp-2019-0006

Barcia, P. L. (2004). Los diccionarios del español de la Argentina. Academia Argentina de Letras.

Bermúdez, W., y Bermúdez, S. (s. f.). Lenguaje del Río de la Plata. Inédito.

Bértola, C. (2013). Filólogos naturalistas en la Banda Oriental en los siglos XVIII y XIX: estudio lingüistico comparativo entre el legado de un naturalista demarcador y el de un demarcador naturalista [Monografía de grado, Universidad de la República].

Cabakian, A. (16-17 de octubre de 2012). Los glosarios: un capitulo de nuestra historia lexicográfica. VI Seminario sobre lexicología y lexicografía del español y del portugués americanos, Montevideo, Uruguay. http://www.academiadeletras.gub.uy/innovaportal/file/102864/1/cabakian.pdf

Casares, J. (1942). Diccionario ideológico de la lengua española. Gustavo Gilli S. A.

Chans, R., y Urse, J. C. (13 de octubre de 2011). Léxico histórico en Juan Manuel Pérez Castellano: estudio del vocablo "citrino". V Seminario sobre lexicología y lexicografía del español y del portugués americanos: a 200 años del inicio del proceso independentista del Uruguay, Montevideo, Uruguay. http://www.academiadeletras.gub.uy/ innovaportal/file/102848/1/chans-urse.pdf

Chans, R., y Urse, J. C. (16-17 de octubre de 2012). Léxico histórico en José Manuel Pérez Castellano: cachinga (¿cachimba?). VI Seminario sobre lexicología y lexicografía del español y del portugués americanos, Montevideo, Uruguay. http://www.academiadeletras.gub.uy/innovaportal/file/102863/1/chans-urse.pdf

Coll, M. (2012). Léxico de origen indígena y africano en dos escritores montevideanos de principios del siglo XIX: la mirada de José M. Pérez Castellano y Dámaso A. Larrañaga. Stockholm Review of Latin American Studies, 8, 49-64. https://www.lai.su.se/polopoly fs/1.135181.1368788565!/menu/standard/file/SROLAS 08 2012.pdf

Coll, M. (2013a). Prácticas lexicográficas del siglo XIX en territorio uruguayo: de la nominación al registro de piezas de museo. Revista Argentina de Historiografía Lingüística, 2, 115-136.

Coll, M. (2013b). Representation of Charrúa Speech in 19th Century Uruguayan Literature. En L. Callahan (Ed.), Spanish and Portuguese Across Time, Place, and Borders. Studies in Honour of Milton M. Azevedo (pp. 110-131). Palgrave Macmillan. 


\section{SECCIÓN: LEXICOGRAFÍA \\ LA PRODUCCIÓN LEXICOGRÁFICA DE FINES DEL SIGLO XX \\ EN URUGUAY \\ Magdalena Coll y Clara Pérez Pucci}

Coll, M. (2015). Prácticas lexicográficas e ideas lingüísticas en Benjamín Fernández y Medina. Anuario de Letras, 1, 53-85. https://doi.org/10.19130/iifl.adel.3.1.2015.71

Coll, M. (2017). Hacia una periodización de la lexicografía en el territorio uruguayo. Lingüística, 33(1), 47-71. https://doi.org/10.5935/2079-312X.20170004

Coll, M. (2018). Prólogo a El léxico de los uruguayos y otros estudios, de Adolfo Berro García (pp. 7-63). Colección de clásicos uruguayos. Ministerio de Educación y Cultura.

Coll, M., y Montenegro, Y. (16-17 de octubre de 2012). Colección de glosarios rioplatenses del siglo XIX: organización y disponibilización en línea. VI Seminario sobre lexicología y lexicografía del español y del portugués americanos, Montevideo, Uruguay.

CVBMIDUED = Tálice, R. (1993). Cien vocablos biológicos o médicos imperfectamente definidos en la última edición del DRAE (1992). Inédito.

Da Rosa, J. J., y Lucián, E. (2016). Lenguaje del Río de La Plata: rescate de un diccionario dialectal desconocido. Thesaurus, 58, 128-154.

DDVUAEMP = Alberti, E., Berro, M., Mieres, C., y Miranda, E. (1971). Diccionario documentado de voces uruguayas en Amorim, Espinola, Mas de Ayala, Porta. Universidad de la República.

De Granda, G. (1968). Reseña a Lengua-Literatura-Folklore: Estudios dedicados a Rodolfo Oroz [Facultad de Filosofía y Educación, Universidad de Chile, 1967]. Thesaurus, 23(1), 96.

DUD $=$ Mieres, C., Miranda, É., Alberti, E., y Berro, M. (1966). Diccionario uruguayo documentado. LIGU.

Elizaincín, A. (2006). Sobre la lexicografía como ejercicio intelectual. El caso de Washington y Sergio Bermúdez y su Lenguaje del Río de la Plata. En C. Company Company (Ed.), El español en América. Diatopía, diacronía e historiografía. Homenaje a José G. Moreno de Alba (pp. 547-559). Universidad Nacional Autónoma de México.

Enguita Utrilla, J. (2012). Trasfondo léxico rioplatense en la obra americana del naturalista aragonés Félix de Azara. Revista Internacional de Lingüística Iberoamericana, 10(20), 51-70.

Fajardo Aguirre, A. (2010). La lexicografía del español de América. Parte primera: evolución, tipología y metodologías. En M. Aleza y J. Enguita (Coords.), La lengua española en América: normas y usos actuales (pp. 317-354). Universidad de Valencia.

Fernández Guerra, A. (2012). Presencia de vocablos de origen africano en el Lenguaje del Río de la Plata. En L. Álvarez López y M. Coll (Eds.), Una historia sin fronteras: léxico de origen africano en Uruguay y Brasil (pp. 97-118). Acta Universitatis Stockolmiensis, Romanica Stockholmiensa, 30.

Granada, D. (1889). Vocabulario rioplatense razonado. Elzeviriana.

Guarnieri, J. C. (1979). Diccionario del lenguaje rioplatense. Banda Oriental.

Kornfeld, L., y Kuguel, I. (1999). Tratamiento de los indigenismos y representación de las lenguas indígenas en la lexicografía monolingüe argentina del siglo XIX. En E. Narvaja 


\section{SECCIÓN: LEXICOGRAFÍA \\ LA PRODUCCIÓN LEXICOGRÁFICA DE FINES DEL SIGLO XX EN URUGUAY \\ Magdalena Coll y Clara Pérez Pucci}

de Arnoux y R. Bein (Comps.), Prácticas y representaciones del lenguaje (pp. 65-74). Eudeba.

Kühl de Mones, U. (1986). Los inicios de la lexicografía del español del Uruguay. El Vocabulario rioplatense razonado por Daniel Granada (1889-1890). Lexicographica. Series Maior, 8. https://doi.org/10.1515/9783111341774

Kühl de Mones, U. (1993). Nuevo diccionario de uruguayismos. En G. Haensch y R. Werner (Dirs.), Nuevo diccionario de americanismos. Volumen 3. Instituto Caro y Cuervo.

Kühl de Mones, U. (1997). Azara como fuente lexicográfica. En Á. Mones y A. Klappenbach (Eds.), Un ilustrado aragonés en el Virreinato del Río de la Plata: Félix de Azara (1742-1821). Estudios sobre su vida, su obra y su pensamiento, Anales del Museo Histórico Natural, 2. ${ }^{\mathrm{a}}$ serie, IX, 61-67.

Kühl de Mones, U. (1998). "Introducción" y edición al Diccionario rioplatense razonado, de Daniel Granada. Arco Libros.

Laguarda Trías, R. (1969). Afronegrismos rioplatenses. Boletín de la Real Academia Española, 49, 27-116.

Lauria, D. (2010). Lengua y nación en la lexicografía argentina de fines del siglo XIx y principios del xx: análisis de los mecanismos de ejemplificación y citación. Res Diachronicae, 8, 47-70.

Lauria, D. (2012). Continuidades y discontinuidades de la producción lexicográfica del español de la Argentina. Un análisis glotopolítico de los diccionarios publicados en el marco del Centenario y en el del Bicentenario de la Revolución de Mayo [Tesis doctoral, Universidad de Buenos Aires].

López, C. (2017). La lexicografía uruguaya a fines del siglo XIX: una mirada a través de los diccionarios de Daniel Granada y de Wáshington y Sergio Bermúdez [Tesis de maestría, Universidad de la República].

Malaret, A. (1946). Diccionario de americanismos. Emecé.

Mieres, C., Miranda, É., Berro, M., y Alberti, E. (1973). Comentarios a la última edición del Diccionario de la Real Academia Española (1970). Boletín de la Academia Nacional de Letras, 1(2), 7-49.

Montenegro, Y. (2019). Historia de la lexicografía en el Uruguay: el Lenguaje del Río de la Plata de Wáshington y Sergio Bermúdez [Tesis de maestría, Universidad de la República].

Pereda Valdés, I. (1965). El negro en el Uruguay. Pasado y presente. Revista del Instituto Histórico y Geográfico del Uruguay, 25, 181-185.

Pereira Rodríguez, J. (1961). El Lenguaje del Río de la Plata, de Sergio Wáshington Bermúdez. Inter-American Review of Bibliography, 15(1), 230-233.

Real Academia Española (1950). Diccionario manual e ilustrado de la lengua española. Espasa-Calpe.

Real Academia Española (1956). Diccionario de la lengua española (18. a edición).

Real Academia Española (1970). Diccionario de la lengua española (19. a edición). 
Real Academia Española (1992). Diccionario de la lengua española (21. a edición).

Rosell, A. (1978). El diccionario de los Bermúdez. Boletín de la Academia Nacional de Letras, 2. 'ápoca, 6, 13-38.

Soca, R. (2017). Entre el arrabal y el rancherio: el Diccionario del lenguaje rioplatense de Juan Carlos Guarnieri (1979) [Tesis de maestría, Universidad de la República].

VDPLEU = Argüello, C. (1963). Vocabulario documentado en la producción literaria de escritores uruguayos. Inédito.

$V I=$ Alba, L., Jones, C. y Da Rosa, J. J. (1985). La vestimenta del Uruguay en el siglo XX. Glosario. Inédito.

$V U=$ Berro García, A. (1967). Uruguayismos en el habla común. En G. Carrillo Herrera (Ed.), Lengua, literatura, folklore. Estudios dedicados a Rodolfo Oroz (pp. 53-80). Ed. Universitaria.

Zimmermann, K. (2003). El fin de los diccionarios de americanismos. La situación de la lexicografía del español de América después de la publicación de los "Diccionarios contrastivos del español de América”. Revista Internacional de Lingüística Iberoamericana, 1(1), 71-83. 